\title{
Nondestructive Investigation of Stress-Induced Damage in Concrete
}

\author{
P. Shokouhi, A. Zoëga, and H. Wiggenhauser \\ Non-Destructive Damage Assessment and Environmental Measurements Methods Department, \\ BAM Federal Institute for Material Research and Testing, 12205 Berlin, Germany
}

Correspondence should be addressed to P. Shokouhi, parisa.shokouhi@bam.de

Received 1 December 2009; Revised 8 March 2010; Accepted 25 April 2010

Academic Editor: Patrice Rivard

Copyright ( $\odot 2010$ P. Shokouhi et al. This is an open access article distributed under the Creative Commons Attribution License, which permits unrestricted use, distribution, and reproduction in any medium, provided the original work is properly cited.

The changes in the sonic surface wave velocity of concrete under stress were investigated in this paper. Surface wave velocities at sonic frequency range were measured on a prismatic concrete specimen undergoing several cycles of uniaxial compression. The loading was applied (or removed) gradually in predefined small steps (stress-controlled). The surface wave velocity was measured at every load step during both loading and unloading phases. Acoustic Emission (AE) test was conducted simultaneously to monitor the microcracking activities at different levels of loading. It was found that the sonic surface wave velocity is highly stress dependent and the velocity-stress relationship follows a particular trend. The observed trend could be explained by a combination of acoustoelasticity and microcracking theories, each valid over a certain range of applied stresses. Having measured the velocities while unloading, when the material suffers no further damage, the effect of stress and damage could be differentiated. The slope of the velocity-stress curves over the elastic region was calculated for different load cycles. This quantity was normalized to yield a dimensionless nonlinear parameter. This parameter generally increases with the level of induced damage in concrete.

\section{Introduction}

To ensure the integrity of an existing structure, one needs to have a reliable estimation of in-situ material properties, the remaining strength and relevant damages in the loadcarrying components. Nondestructive testing (NDT) techniques which can provide a reliable assessment of one or more of these parameters, without harming the structure itself, are invaluable to inspectors and engineers. Among the applicable NDT methods, acoustic techniques have long been used for inspection of concrete structures, both in defect detection and material characterization applications. When used for defect detection, acoustic techniques are especially powerful tools for locating the defects, which introduce an impedance discontinuity within the concrete structure (e.g., voids, cracks, and flaws). In applications concerning material characterization, their advantage lies in their ability to give a direct estimation of mechanical material properties through measuring the acoustic wave velocities.

The results of acoustic tests are usually analyzed and interpreted based on the theory of elastic wave propagations in linearly elastic homogenous solids. According to this theory, the velocity of acoustic waves propagating in linear elastic materials is a function of elastic material properties (i.e., Lame's or 2nd order elastic constants) and mass density. For example, the compressional wave ( $P$-wave) velocity in a linear elastic rod is given by

$$
V_{P}=\sqrt{\frac{\lambda+2 \mu}{\rho_{0}}} .
$$

Therefore, given the mass density $\rho_{0}$, by measuring the velocity $V_{P}$, one can directly obtain the elastic properties $\lambda, \mu$. In the case of a composite material such as concrete, many of the assumptions behind this formulation cease to be valid. Concrete is both heterogonous and anisotropic and exhibits nonlinear stress-strain behavior. However, the use of this formulation is still justified. The reason lies in the particular characteristics of acoustic tests tuned to concrete inspection applications. On one hand, (in order to avoid scattering and attenuation) the range of test frequencies $(<50 \mathrm{KHz})$ is chosen such that the incident wave is "blind" 
to the concrete heterogeneities. On the other hand, the level of stress during the acoustic test is too low to result in any significant nonlinearity in material's behavior. The latter argument is invalid in the case of in-situ field investigation, when the concrete structural component under investigation is simultaneously subjected to stresses much larger than those applied during the acoustic test. As it will be shown here, in such cases, the velocities depend not only on the material properties, but also on the level of stress (and internal damage) within the component. The stress dependency of elastic wave velocities is not currently taken into account when concrete structures are inspected by acoustic techniques.

It has been shown both theoretically and experimentally that the state of stress in nonlinear materials will induce anisotropy in their elastic properties [1]. As a result, the propagation velocities of waves in an elastic medium subjected to large deformation-compared to particle movements in the acoustic test-are stress dependent. The changes in the propagation velocities of ultrasonic waves due to the state of strain (or stress) in an elastic solid are generally referred to as acoustoelastic effects. Acoustoelastic effects have long been investigated in numerous studies concerning metallic substances [1-3]. Only very recently, this effect has been measured in concrete under low levels of stress [4-6].

In a brittle crack-prone medium such as concrete, acoustoelastic effects are not be the sole reason behind the stress-dependent material properties (especially at higher stress levels). In the case of rocks, it has been demonstrated that the formation, growth, and coalescence of microcracks at different stress levels introduce anisotropy in ultrasonic wave velocities [7]. When the loading is anisotropic, the changes in ultrasonic wave velocities depend not only on the level of induced stress but also on the direction of stress relative to the direction of propagation and/or polarization of the ultrasonic waves. The direction-dependency of ultrasonic wave velocities has been demonstrated for metals [1], rocks [7] as well as concrete $[4,6]$.

Load-carrying concrete structural components experience different levels of stress and strain over the life span of the structure. It is believed that acoustoelasticity and microcracking theories together can explain the stress-dependency of elastic properties of concrete, each valid over a certain stress range. At very low stress levels when concrete is still elastic, acoustoelasticity is the dominant effect. At high-stress levels when concrete is not elastic anymore, microcracking is believed to be the dominant cause. Similar arguments have been made in describing the stress dependency of elastic wave velocities in rocks [8].

This paper presents the partial results of an experimental study aimed at investigating the stress and damage dependency of acoustic wave velocities in concrete. In the previously reported studies of similar objectives, the ultrasonic wave velocities of concrete specimens were measured in a through transmission mode and the changes in the velocities or amplitudes of transmitted wave were correlated to the state of internal damage in the specimen. The velocities measured perpendicular to the loading gradually decrease but the decease is not significant until reaching critical stress levels $[9,10]$. When measured parallel to the loading, the velocities slightly increase (not more than 1\%) over the elastic range [4-6]. Reliable measurement of such subtle changes is not typically possible using the conventional time-of-flight measurement techniques and was possible employing correlation-based techniques [4-6].

The study presented here is different from those reported in the literature in two distinct ways. First, instead of ultrasonic wave velocities, the velocities of acoustic waves in the sonic range $(\sim 10 \mathrm{KHz})$ were measured. Second, instead of measuring the velocity of body waves (i.e., shear or compression waves), the surface wave velocities were measured here. As will be demonstrated in this paper, the surface wave velocities measured in the sonic range of frequencies are a few times more sensitive to the level of stress and damage in concrete than ultrasonic body waves. The practical advantage of such measurements is that since the velocities are measured on the surface, only a onesided access to the structure is required. Moreover, the test setup is simple and inexpensive and the data analysis (i.e., velocity measurement) is straightforward. Therefore, such measurements have a great potential to be used for estimating the in-situ state of stress or damage in concrete.

\section{Theory of Acoustoelasticity}

Acoustoelastic theory gives the changes in the elastic wave velocities due to the state of stress in a material exhibiting a nonlinear elastic behavior. For an elastic column of nonlinear constituent material under uniaxial stress (shown in Figure 1), the velocity of longitudinal plane waves propagating in the direction of the applied stress $\left(V_{11}^{\sigma_{1}}\right)$ can be approximated as [11]

$$
\begin{aligned}
& \rho_{0}\left(V_{11}^{\sigma_{1}}\right)^{2} \\
& \quad=\rho_{0}\left(V_{11}^{0}\right)^{2}-\frac{\hat{\sigma}_{1}}{E}[(7 \lambda+14 \mu-6 l)-2 \nu(3 \lambda-6 l-2 m)],
\end{aligned}
$$

where $\rho_{0}$ is the initial density, $\sigma_{1}$ is the applied stress, $V_{11}^{0}$ is the velocity in the stress-free state $\left(=V_{P}\right), \lambda$ and $\mu$ are the second order elastic constants generally known as Lame's constants and finally $l, m$, and $n$ are Murnaghan's third-order elastic constants. Therefore, acoustoelastic theory indicates a linear relationship between the applied uniaxial stress and the squared values of the longitudinal wave velocities. As long as the stress-induced changes are very small, one may use the linear approximation $V_{11}^{\sigma_{1}^{2}}=V_{11}^{0^{2}}+\left(V_{11}^{\sigma_{1}}-V_{11}^{0}\right)\left(2 V_{11}^{0}\right)$. Applying this approximation and substituting the $V_{11}^{0}=$ $V_{P}$ on the right hand by its equivalent expression from 1 , simplifies the above equation to a linear relationship between the relative change in velocity and applied stress;

$$
\begin{gathered}
\frac{V_{11}^{\sigma_{1}}-V_{11}^{0}}{V_{11}^{0}}=\frac{-\hat{\sigma}_{1}}{2 E}\left[\frac{(7 \lambda+14 \mu-6 l)-2 \nu(3 \lambda-6 l-2 m)}{\lambda+2 \mu}\right], \\
\frac{\Delta V_{11}}{V_{11}^{0}}=\frac{-\hat{\sigma}_{1}}{E} \beta_{11},
\end{gathered}
$$




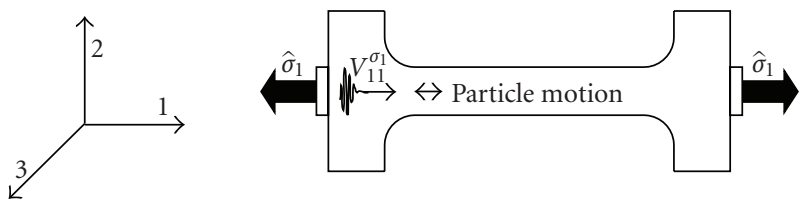

FIgURE 1: Ultrasonic wave propagation in the direction of loading in a uniaxially loaded column. If the constituent material is nonlinear, the propagation wave velocity $V_{11}^{\sigma_{1}}$ is stress dependent.

where $\beta_{11}$ is a dimensionless nonlinear parameter. Considering the relationship between the compression and surface wave velocities $V_{P}$ and $V_{R}$ as a function of Poisson's ratio $v\left(V_{R} \approx V_{P}(\sqrt{1-2 v / 2-2 v} / 1.13-0.16 v)\right)$, it is reasonable to assume that acoustoelastic theory yields a similar relationship between surface wave velocity and the applied stress. More rigorous equations governing the velocity of Rayleigh waves propagating along a surface of a uniaxially loaded column given in other references [2] confirm this assumption.

\section{Microcracking}

Relying on acoustoelasticity theory, the stress dependency of acoustic wave velocities in a preloaded elastic homogeneous body can be quantitatively evaluated. However, this theory can not fully explain the velocity-stress relationship for concrete over the entire range of stresses. The discrepancy lies in the brittle crack-prone heterogeneous nature of concrete. At higher stress levels (typically beyond $30 \%$ of the strength), microcracks start to develop within the concrete mass. Since microcracking is an irreversible (plastic) process, the material soon leaves the elastic region. Therefore, the theory of acoustoelasticity can no longer describe the velocitystress relationship. The initiation, growth, and coalescence of microcracks are believed to play an important role in the observed stress-induced changes in the surface wave velocities at high-stress levels.

The mechanical behavior of brittle material is greatly influenced by the formation and development of cracks. The interaction of elastic waves propagating in a medium containing microcracks depends on the size of the cracks relative to the wavelength of the propagating waves. Elastic waves of wavelengths shorter than the size of the cracks will be reflected from and scattered around the edges of the cracks. On the other hand, if the cracks are comparable in size or smaller than the incident elastic waves, their presence influence the overall elastic properties of the medium. For example, the presence of microcracks generally results in a reduction in the velocities of elastic waves propagating in a medium. The changes in wave velocities depend on the size, orientation (relative to the direction of wave propagation), condition (e.g., empty or filled), and volumetric distribution of cracks. Hudson [12] derived the overall elastic parameters, wave speeds, and attenuation of elastic waves in materials containing circular cracks of different distribution density, orientation (i.e., aligned along a particular direction or randomly oriented) and condition (i.e., empty or filled with fluids or weak materials).

It is reasonable to assume that the orientation and distribution of microcracks are random for a medium in its natural state or under isotropic external pressure. Under anisotropic stress conditions, however, microcracks show some degrees of preferred orientation which in turn, will introduce stress-induced anisotropy in the elastic properties of materials. Such effects have been reported in the studies concerning stress-induced anisotropy in rocks. Sayers et al. [7], measured ultrasonic velocities in three orthogonal directions for a cubic sample of Berea sandstone. He used Hudson's [12] formulas to evaluate the distribution and orientation of microcracks within the sample. The similarities between velocity-stress relationships reported for rocks and those obtained in our experiment suggest that microcracking has a key role in the observed stress-dependency of wave velocities in concrete.

\section{Experimental Investigation}

An experimental study was conducted to investigate the stress and damage-dependency of acoustic wave velocities in concrete. Another objective of this study was to investigate whether the observed effects depend on the stress history of the material (or show stress memory effects).

The general scheme of the experimental series can be described as follows. Prismatic concrete specimens of different sizes and properties (i.e., aggregate size, water to cement ratio, with or without reinforcement, etc.) were cast. After being hardened (at least four weeks after casting), the specimens were instrumented. The instrumentation included at a minimum a hammer and two sensors mounted along a line on one side of specimen, parallel to its largest dimension (length). This basic setup is illustrated schematically in Figure 2.

Each instrumented specimen was placed in the loading machine and compressed uniaxially along its length. The load was applied in predefined small steps (stress-controlled mode). At every load step, the load (and consequently, the stress level) was held constant long enough to allow the measurement of surface wave velocities. With the basic setup of one hammer and two sensors described earlier, the measurement involved an automatic firing of the hammer and recording the response at the two sensors. Once the measurement was completed, the load was raised to the next level and the measurements were repeated at the new load step. Some specimens (like the one discussed here) were subjected to more than one loading cycle to allow study of the stress memory effects. In such cases, the maximum desirable load level at each cycle was determined and the procedure described above was repeated at every load step of every loading cycle. Once the desirable stress level was reached, the specimen was stepwise unloaded. In some of the experiments (like the one presented here), the surface wave velocities were also measured during the unloading steps. At the end of one full cycle, the specimen was brought to the stress-free state. After a short break, the subsequent loading 


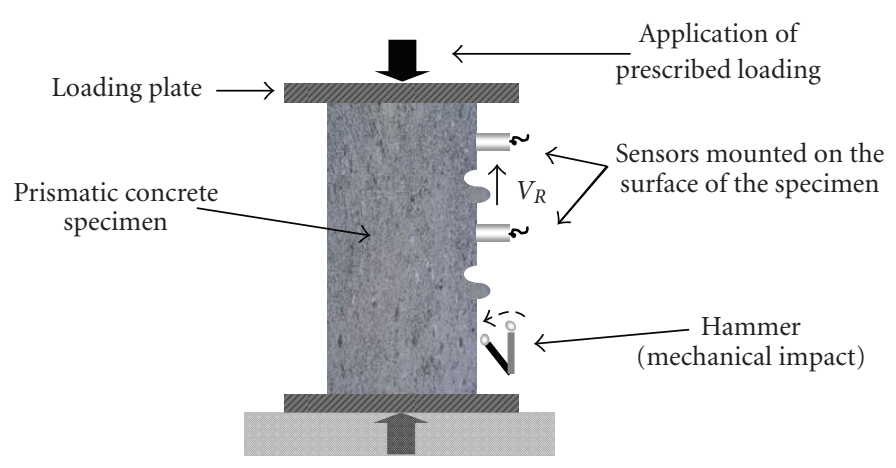

FIgURe 2: The schematics of the basic measurement setup.

cycle was followed. In the last load cycle, the specimen was loaded until reaching failure. To avoid possible damages to the instrumentation, the sensors were usually dismounted at final load steps (close to the failure of the specimen), but the loading was continued until the specimen broke.

One of these experiments is described in detail here. The detailed description of this experiment, followed by the illustration and discussion of its results are provided.

4.1. Specimen. A prismatic $0.2 \times 0.2 \times 0.6 \mathrm{~m}^{3}$ specimen of plain concrete (max. aggregate size of $16 \mathrm{~mm}$, water to cement ratio $(\mathrm{w} / \mathrm{c})=0.55)$ was used for this experiment. The characteristics of the concrete mixture together with some of its mechanical properties are provided in Table 1. The concrete mix was prepared according to the C30/C37 mix design given in the European (Eurocode2) or German guidelines (DIN 1045-1). Five identical specimens of the same batch were cast together in wooden molds. The specimens were stripped of the molds after 5 days and then put in ambient conditions for 28 days before being tested.

4.2. Measurement Setup. The setup for surface wave velocity measurements is shown in Figure 3.

The instrumentation included a small mechanical hammer (from Olson Instruments Inc.) and four sensors (Sensors 1 to 4), from Acsys (ACS Ltd.) all mounted along a line on one side of the specimen. The sensors are dry contact (i.e., they do not require application of a coupling agent) and spring loaded. To ensure sufficient pressure between the sensors and the surface of the specimen over the entire duration of the test, a simple wooden "jacket" was made. As depicted in Figure 3, the jacket consisted of two wooden boards with holes at the designated sensor locations. The boards were fastened together by four metal bars. As a result, the sensors were tightly pressed against the surface of the specimen throughout the test. One of the two boards was cut in the middle to allow mounting of the hammer. The hammer was glued directly on the surface of the specimen. The distance between every two adjacent sensors and that between the hammer and the nearest sensors on either side was chosen as $0.1 \mathrm{~m}$.

The surface wave velocity at every load step was calculated by measuring the surface wave arrival time delays for the Sensor pair $(1,2)$ and $(3,4)$. Five additional sensors (depicted in Figure 3(b)) were mounted on the opposite side of the specimen, to study the stress-induced changes in the frequency content of the body waves travelling through the specimen. The hammer and the sensors were controlled by a computer equipped with three built-in PXI cards from Spectrum (GmbH, Germany). Each card could acquire up to four channels with a sampling rate of $1 \mathrm{MHz}$ and a resolution of 16 bits in the used voltage range of $\pm 2.5 \mathrm{~V}$. Therefore, using this system up to 12 channels (e.g., 9 channels for this particular experiment) could be recorded simultaneously.

In order to monitor the stress-induced microcracking development, a simple Acoustic Emission (AE) test was simultaneously conducted. A simple single-sensor AE test setup was developed in house and used in this experiment. A highly sensitive broad band acceleration sensor (also from Acsys) was employed for this purpose. As seen in Figure 3(b), the sensor was mounted close to the middle of the specimen, opposite to the hammer. The data acquisition included an analog digital converter from Pico with a sampling rate of $625 \mathrm{kHz}$ and a resolution of 12 bits in a range of $\pm 1 \mathrm{~V}$. A certain threshold was set and events exceeding this threshold were fully recorded. The not crackingrelated recorded signals (i.e., noise) were later excluded in postprocessing.

4.3. Loading. The specimen was subjected to nine successive load cycles (loading and unloading). In all the cycles, the pressure was applied gradually, in steps of $50 \mathrm{KN}$ (or 1.25 MPa) until the maximum prescribed level of load was reached (see Table 2). The specimen was then unloaded. The unloading was also gradual, but in larger steps of 100 $\mathrm{KN}$ (or 2.50 MPa). The unloading was continued until the specimen was fully unloaded (stress-free). The measurement setup was checked while the specimen was at rest. The new load cycle then followed. In load cycle 9, the instrumentation was detached after reaching $86 \%$ of the load at failure and the loading continued until the specimen broke. The maximum load carried by the specimen before it failed was recorded as the load at failure.

The loading was applied using a 2-MN loading machine. Due to a loading error (i.e., a sudden jump in the loading 
TABLE 1: Mixture proportions and some mechanical properties of concrete mixture.

\begin{tabular}{|c|c|c|c|c|c|c|c|c|}
\hline \multirow{2}{*}{$\begin{array}{l}\text { Sample } \\
\text { Designation }\end{array}$} & \multirow{2}{*}{$\begin{array}{l}\text { Mixture } \\
\text { Proportions } \\
\text { C:CA : FA }(\mathrm{w} / \mathrm{c})^{*}\end{array}$} & \multicolumn{6}{|c|}{ Aggregate Proportions } & \multirow{2}{*}{$\begin{array}{l}\text { Compressive } \\
\text { Strength } \\
(\mathrm{MPa} / \mathrm{ksi})\end{array}$} \\
\hline & & $\begin{array}{c}5 / 16^{\prime \prime} \\
(8 \mathrm{~mm})^{* *}\end{array}$ & No. 5 (4 mm) & $\begin{array}{l}\text { No. } 10 \\
(2 \mathrm{~mm})\end{array}$ & $\begin{array}{l}\text { No. } 18 \\
(1 \mathrm{~mm})\end{array}$ & $\begin{array}{c}\text { No. } 35 \\
(0.5 \mathrm{~mm})\end{array}$ & $\begin{array}{c}\text { No. } 50 \\
(0.3 \mathrm{~mm})\end{array}$ & \\
\hline \multirow{2}{*}{$\begin{array}{l}\text { Test Specimen } \\
\text { (C30/C37) }\end{array}$} & \multirow{2}{*}{$1: 2.32: 2.87(0.55)$} & \multicolumn{2}{|c|}{ Coarse (CA) } & \multicolumn{5}{|c|}{ Fine (FA) } \\
\hline & & $25 \%$ & $20 \%$ & $14 \%$ & $10 \%$ & $11 \%$ & $21 \%$ & $42.8 / 6.2$ \\
\hline
\end{tabular}

${ }^{*}$ Fractions in weight: C: Cement (Type I), CA: Coarse Aggregates, FA: Fine Aggregates, and (w/c): water to cement ratio.

** The maximum aggregate size was $5 / 8^{\prime \prime}$ or $16 \mathrm{~mm}$.

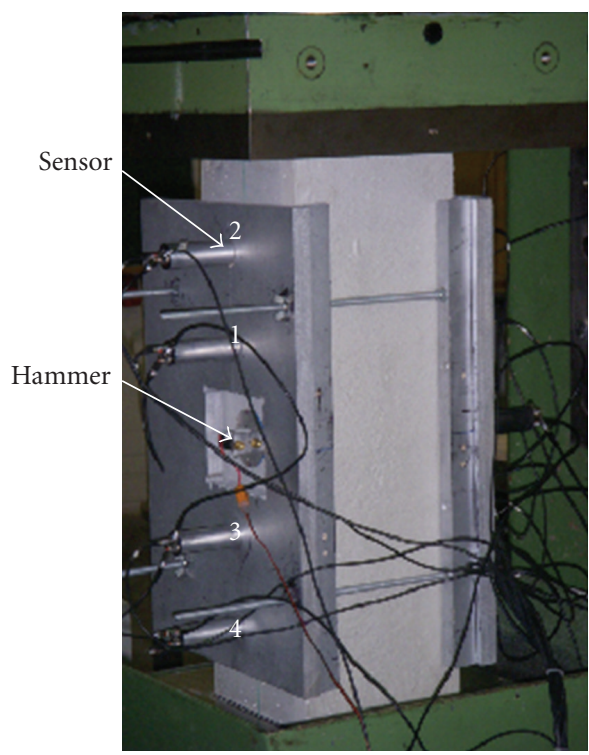

(a)

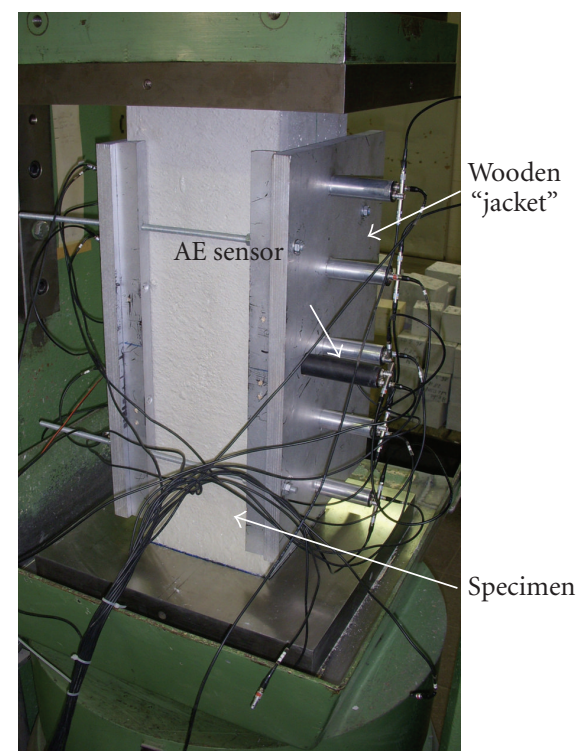

(b)

FIGURE 3: Measurement setup: (a) the sensor arrangements for surface wave velocity measurements, (b) the five extra sensors on the opposite side of the specimen and the additional AE sensor.

instead of a stepwise increase) in the first loading cycle, the results of the measurements made during this cycle were not reliable and therefore, are not included in the subsequent illustrations and discussions.

4.4. Measurement Procedure. In every load cycle, the first measurements were taken before the application of the load, at the stress-free state. Before raising the load to the next designated load level, the AE test was run. This was done mainly to record the inevitable noise from the loading machine, so that its characteristics could be studied and be used later in separating noise from the event signal at later load steps. After reaching the desired load level, the $\mathrm{AE}$ test was active until no more events were registered (over a certain period of time). Then, the AE test was "turned off" and the hammer was fired and the signals were recorded simultaneously at all nine sensors. At every load step, the measurements were repeated 36 times to ensure repeatability and reliability of the collected data. Once all 36 measurements were completed, the AE test was "turned on" again and the load was raised to the next level. The exact same procedure was followed during both the loading and unloading phases.

\section{Experimental Results and Discussions}

The results of the surface wave velocity measurements together with those obtained from the AE test are illustrated and discussed in this section. Although only the results of one experiment are discussed here, in drawing the conclusions, occasional references have been made to the outcome of our other experiments published elsewhere [13-15].

5.1. Stress Dependency of Sonic Surface Wave Velocities. The results of the surface wave velocities measurements made during this experiment clearly reveal the surface wave velocities (measured parallel to the loading axis) are stress dependent. To demonstrate the typically obtained velocitystress relationship, the measurements taken during the 8th load cycle of this test are singled out and discussed here. During this cycle, the specimen was reloaded stepwise from the stress-free (or zero-load) state until reaching 71 percent of the final load at failure. Only the results of the measurement taken during the loading are discussed in this section.

The relative changes in velocity $\Delta V / V_{0}=\left(V-V_{0}\right) / V_{0}$ versus stress measured during the 8 th loading cycle are 
TABLE 2: The maximum level of applied load in each cycle in terms of load, stress, and percentage of the load at failure.

\begin{tabular}{lccccccccc}
\hline Cycle & 1 & 2 & 3 & 4 & 5 & 6 & 7 & 8 & 9 \\
\hline Load $(\mathrm{MN})$ & 0.15 & 0.30 & 0.50 & 0.30 & 0.60 & 0.80 & 0.55 & 0.95 & 1.15 \\
Stress (MPa) & 3.75 & 7.50 & 12.50 & 7.50 & 15.00 & 20.00 & 13.75 & 23.75 & 28.75 \\
\% Load at Failure & 11 & 23 & 38 & 23 & 45 & 60 & 41 & 71 & 86 \\
\hline
\end{tabular}

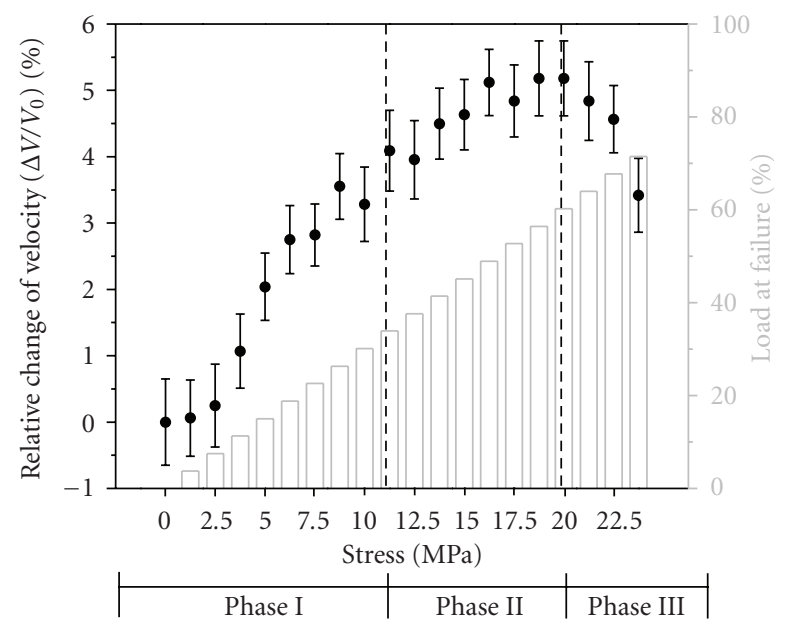

FIGURE 4: Surface wave velocity versus stress in the 8th load cycle. The velocities are normalized with respect to the velocity measured at the stress-free state of this load cycle. The bars indicate the percentage of load at failure at each load step.

shown in Figure 4. The velocities are normalized here with respect to $V_{0}$, the velocity obtained at the zero-load state in the beginning of this load cycle. The circles show the mean values obtained by averaging the arrival times of all 36 repeated measurements at every load cycle. The error bars indicate one standard deviation of uncertainty.

The surface wave velocity-stress relationship shown in Figure 4 is typical for this type of measurements. We have observed the same general trend in our other experiments on specimens of different sizes and types. The velocity-stress curve can be broken into three phases.

(i) Phase I (at stress levels up to about $30-35 \%$ of Load at Failure), where the velocity increase with stress. In this phase, concrete can be still regarded as elastic and the acoustoelastic effects largely determine the material response. From the microscopic point of view, we believe that this increase is a manifestation of the closure of microcracks lying perpendicular to the load. At this load steps, the stress is high enough to gradually close these microcracks but not so high to cause major damage (new microcracks) within concrete.

(ii) Phase II is recognized by a change in the slope of the velocity-stress curve. Where exactly this change occurs is a signature effect of the stress history of the material (this point will be discussed later in this section). In general terms, the change of slope occurs when the material leaves the elastic zone and suffers plastic damage in the form of extended or new microcracks. This phase ends after the velocities reach a maximum value, after which, the velocitystress relationship enters Phase III.

(iii) During Phase III, the velocities start to decrease at an increasing rate. This is when the microcracks parallel to the loading axis gradually develop to macro- and visible cracks and result in the ultimate failure of the specimen.

As it can be seen in Figure 4, the relative velocity changes are more than $5 \%$ as the stress level is raised to about $70 \%$ of the Load at Failure. As mentioned earlier, this general trend is typical and has been observed in our other experiments on specimens of other sizes and mixtures. In experiments on larger specimens, the uncertainties in measurements are lower (i.e., smaller error bars). It should be emphasized that the velocities here are measured parallel to the loading axis. Other experiments have indicated that the surface wave velocity-stress relationship is also direction dependant. The maximum increase in velocities occur in the direction of the loading, while the largest drop in values at later load steps is observed in the measurements taken perpendicular to the loading [13].

5.2. The "Stress Memory" of Wave Velocities. Having measured the velocities over 9 cycles, we could study the possible "stress memory" effect in the measured wave velocities. According to Kaiser Effect, new microcracks will not develop in a (stable) medium until the stress levels reach beyond the maximum level in the stress history of the medium. This effect can be clearly observed in the results of our simple AE test, depicted in Figure 5(a). The number of registered Events (the blue line in the figure) sharply increases once the previous maximum stress level is reached. During the unloading phases, only a very small number of Events have been registered. These observations are remarkable considering the difficulty in differentiating noise (coming mainly from the loading machine) from signal (or AE Event) and also the fact that our AE test setup included only one single sensor. It should be also noted that the top face of the specimen was considerably rough. The interaction of this roughness with the loading plate at the starting load steps explains the irregularities (i.e., an unexpectedly high number of events) observed in our AE test results, especially at earlier load steps. Otherwise, the test produced satisfactory results in close agreement with Kaiser Effect. The remaining question is, considering the crucial role of microcracking in describing velocity-stress relationship, whether the Kaiser Effect can be also "seen" in surface wave velocity measurements. 


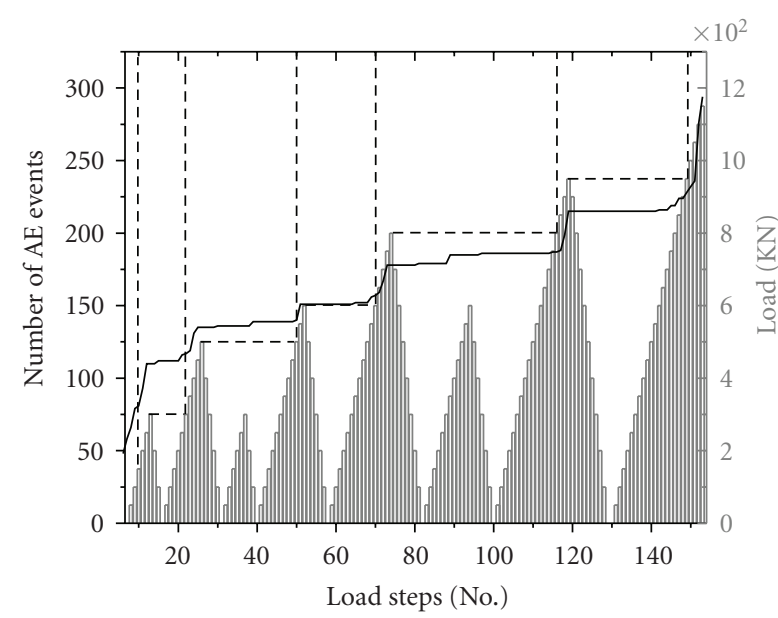

(a)

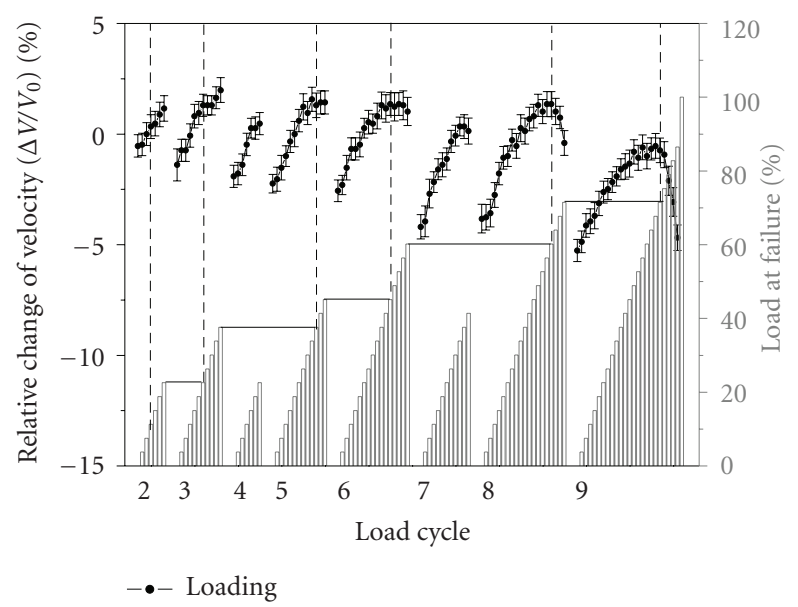

(b)

FIGURE 5: Stress memory effect in surface wave velocity measurements: (a) AE test results and (b) surface wave velocities measured during load cycles 2 to 9 . The second axs in both (a) and (b) indicate the level of loading in $\mathrm{KN}$ and as a percentage of the final load at failure, respectively. The results of the first load cycle are not included in these illustrations.

The surface wave velocities measured during loading phase of load cycles 2 to 9 are plotted together in Figure 5(b). The results of the first load cycle are not included here, because of some measurement errors occurred during this cycle. In calculation of the relative velocity changes $\Delta V / V_{0}=$ $\left(V-V_{0}\right) / V_{0}, V_{0}$ is the surface wave velocity measured at the virgin state, that is before the specimen was loaded. The dashed lines mark the load steps, where the previously applied load level is exceeded. Wherever the maximum level in the stress history of the specimen is exceeded, there is a change in the slope of velocity-stress curve. In another words, the measurements show a stress memory effect. This effect is more obvious in the early loading cycles where the previous loads were not high enough to induce plastic damage and consequently a change of slope.

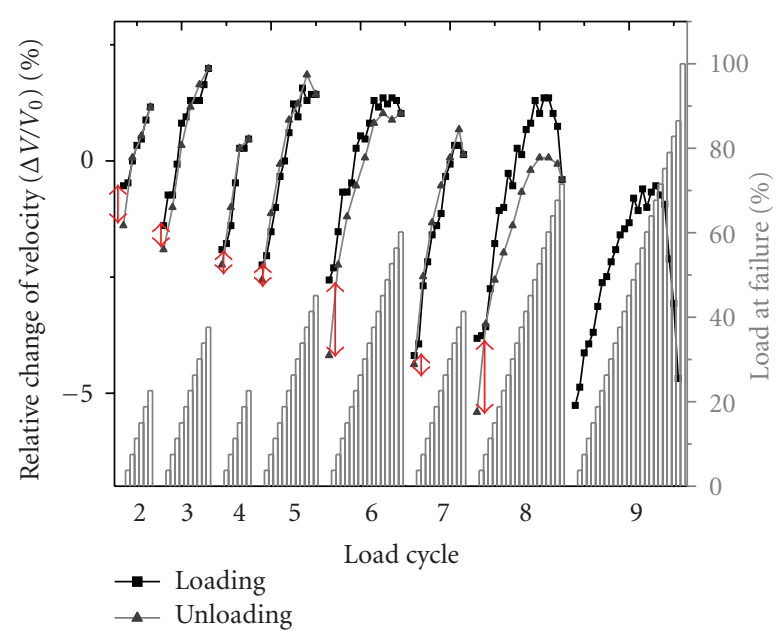

FIGURE 6: Surface wave velocity measurements during both the loading and unloading phases of load cycles 2 to 9 . The arrows mark the average velocity difference at the stress-free state before and after the application of load in each cycle. The second axis indicates the level of loading as a percentage of the final load at failure.

5.3. The Effect of Irrecoverable Damage on Velocity-Stress Relationship. Stress-induced irrecoverable (or plastic) damages in concrete are associated with the development of microcracks. In concrete undergoing gradually increasing axial compression, new microcracks initiate beyond certain stress levels. The majority of the new microcracks develop parallel (or nearly parallel) to the direction of the loading. Concrete containing a considerable amount of microcracks can not be considered elastic anymore and therefore, the theory of acoustoelasticity can no longer describe its velocitystress behavior.

The presence of microcracks results in a decrease in the measured wave velocities. The amount of decrease depends on several factors including the density and orientation of microcracks (with respect to the measurement direction). The effect of microcracking on the surface wave velocities can be demonstrated by comparing the velocity-stress curves measured over loading and unloading phases of various laod cycles. Since no significant additional damage occurs during the unloading phase of a load cycle (Kaiser Effect), the changes in the measured wave velocities over the unloading phase can be only due to the effect of stress. Therefore, the deviation of the velocities during unloading from those measured during the loading phase is due to load-induced irrecoverable damages.

The velocities measured during the loading and unloading phases are superimposed and shown together in Figure 6. To increase the readability of the diagram, the error bars have not been shown in this figure.

It can be observed that, the difference between the measurements during the loading and unloading phases (of one load cycle) becomes significant first in load cycle 6 , where $60 \%$ of the load at failure was reached. As expected, this difference is even larger for load cycle 8 , where $72 \%$ of the load at failure was reached. 
Another important observation in Figure 6 is the difference in the velocities measured on the stress-free specimen before and after each load cycle. These differences are marked with vertical arrows in this figure. With the exception of load cycle 2, this difference is correlated to the level of induced damage in each load cycle: the higher the level of expected damage, the larger is the difference. In another words, as long as the concrete member is not loaded, a lower surface wave velocity is an indication of internal damage and loss of strength.

Finally, the slope of the velocity-stress curves measured over the first few loading or reloading steps (Phase I) was observed to increase with an increase in the stress-induced damage. This observation motivated calculation of a parameter $\beta^{*}$ similar to the dimensionless non-linear parameter $\beta_{11}$. Recalling and rearranging (3), $\beta_{11}$ is calculated using the following equation:

$$
\beta_{11}=\frac{-E}{\hat{\sigma}_{1}} \frac{\Delta V_{11}}{V_{P}}
$$

To obtain $\beta^{*}$, we use the measured relative changes in surface wave velocities (in Phase I) instead of the ultrasonic longitudinal wave velocities propagating in the direction of loading.

$$
\beta^{*}=\frac{-E}{\hat{\sigma}_{1}} \frac{\Delta V}{V_{0}}=-E\left[\frac{\Delta V}{V_{0}} / \widehat{\sigma}_{1}\right]
$$

where the term in brackets is the slope of the relative change of surface wave velocity-stress curve.

Equation (5) was used to calculate $\beta^{*}$ for different load cycles over a stress range of 0 to $7.5 \mathrm{MPa}$ (up to $23 \%$ of load at failure), where concrete can be assumed elastic. The average values of $\Delta V / V_{0}$ measured during the loading (i.e., re-loading) phases of each cycle were used in the calculations. As an example, the calculation of $\beta^{*}$ for the second load cycle is illustrated in Figure 7. The same procedure is repeated for the subsequent load cycles and the resulting average values for $\beta^{*}$ are summarized in Table 2 . The values of $\beta^{*}$ reported here are in the range of $\beta$ values obtained from ultrasonic investigations $[4,5]$. Small differences in the measured values were expected since different material and measurement methods were used here.

A study of $\beta^{*}$ values obtained for load cycles 2 to 9 indicates that this parameter generally increases with the level of induced-damage in concrete. $\beta^{*}$ values were calculated for velocities measured at the beginning of the reloading phase of each load cycle, where the material is still elastic and no irrecoverable damage is expected to occur. Therefore, the value of $\beta^{*}$ measured during the $n$th load cycle reflects the material property at the end of the previous cycle, load cycle $n-1$. According to our measurement results, when concrete undergoes irrecoverable damage in one cycle, a higher average value of $\beta^{*}$ is usually obtained in the subsequent load cycle. The high jump in the value of $\beta^{*}$ in load cycle 7 is particularly remarkable. The last load cycle (cycle 9) is an exception. Despite loading until 71\% in the preceding load cycle (cycle 8 ,) a lower value for $\beta^{*}$ was measured. It can be concluded that, a higher value of $\beta^{*}$

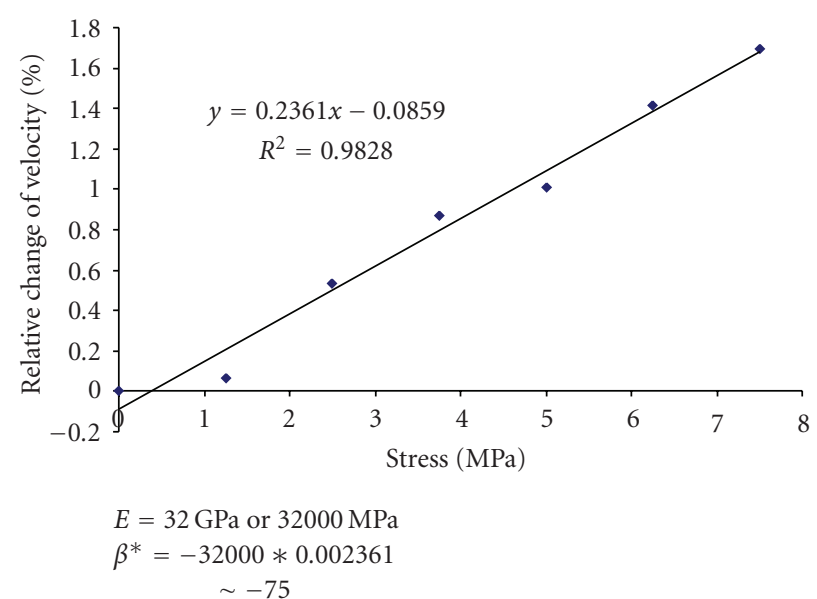

FIgURE 7: Calculation of non-linear parameter $\beta^{*}$ for load cycle 2. The average values of velocity measurements over a low stress range of 0 to $7.5 \mathrm{MPa}$ ( $23 \%$ of load at failure) were used in the calculations.

TABLE 3: The average values of dimensionless nonlinear parameter $\beta^{*}$ for different load cycles.

\begin{tabular}{lcccccccc}
\hline Load Cycle & 2 & 3 & 4 & 5 & 6 & 7 & 8 & 9 \\
\hline$\beta^{*}$ & -75 & -119 & -118 & -118 & -126 & -139 & -143 & -108 \\
\hline
\end{tabular}

measured over low-stress levels is an indication of stressinduced damage, provided that the stress does not exceed $60 \%$ of the strength. Further investigations are currently underway to establish the relationship between the measured $\beta^{*}$ and the state of stress-induced damage in concrete.

\section{Conclusions}

The surface wave velocities measured parallel to the loading on one side of a prismatic specimen undergoing uniaxial compression are highly stress dependent and the velocitystress relationship follows a general multiphase trend. Moreover, the velocity-stress relationship preserves the signature of the loading history of the specimen. By measuring the velocities during unloading phases of load cycles, when no additional damage occurs (according to Kaiser Effect), one can separate the effect of stress and damage on the measured velocities. The dimensionless non-linear parameter $\beta$ was estimated from the measured relative velocity values based on formulations governing the acoustoelastic effects. This parameter generally increases with the level of stress-induced damage.

\section{Acknowledgments}

The authors wish to thank their colleagues at BAM VII.1 for their help in operating the loading machine. This paper was supported in great part by Alexander von Humboldt $(\mathrm{AvH})$ Foundation. This support is greatly acknowledged. 


\section{References}

[1] V. Hauk, Structural and Residual Stress Analysis by Nondestructive Methods: Evaluation-Application-Assessment, Elsevier Science B.V., Amsterdam, The Netherlands, 1997.

[2] F. G. Makhort, O. I. Gushcha, and A. A. Chernoochenko, "On the relations governing rayleigh wave propagation in bodies with initial stresses," International Applied Mechanics, vol. 29, no. 11, pp. 915-920, 1993.

[3] R. H. Bergman and R. A. Shahbender, "Effect of statically applied stresses on the velocity of propagation of ultrasonic waves," Journal of Applied Physics, vol. 29, no. 12, pp. 17361738, 1958.

[4] C. Payan, V. Garnier, J. Moysan, and P. A. Johnson, "Determination of third order elastic constants in a complex solid applying coda wave interferometry," Applied Physics Letters, vol. 94, no. 1, Article ID 011904, 2009.

[5] E. Larose and S. Hall, "Monitoring stress related velocity variation in concrete with a $2 \times 10^{-5}$ relative resolution using diffuse ultrasound," Journal of the Acoustical Society of America, vol. 125, no. 4, pp. 1853-1856, 2009.

[6] Chaix J.-F., I. Lillamand, M.-A. Ploix, V. Garnier, and G. Corneloup, "Study of acoustoelasticity bahavior of concrete material under uniaxial compression," in Proceedings of the Acoustics, pp. 6267-6272, Paris, France, June 2008.

[7] C. M. Sayers, J. G. Van Munster, and M. S. King, "Stressinduced ultrasonic anisotrophy in Berea sandstone," International Journal of Rock Mechanics and Mining Sciences, vol. 27, no. 5, pp. 429-436, 1990.

[8] X. J. Huang, D. R. Burns, and M. N. Toksöz, "The effect of stresses on the sound velocity in rocks: theory of acoustoelasticity and experimental measurements," 2000, http://eaps.mit.edu/erl/research/report1/pdf/huang.pdf.

[9] W. Suaris and V. Fernando, "Detection of crack growth in concrete from ultrasonic intensity measurements," Materials and Structures, vol. 20, no. 3, pp. 214-220, 1987.

[10] C. L. Nogueira and K. J. Willam, "Ultrasonic testing of damage in concrete under uniaxial compression," ACI Materials Journal, vol. 98, no. 3, pp. 265-275, 2001.

[11] S. Takahashi and R. Motegi, "Stress dependency on ultrasonic wave propagation velocity-part 1 . Analysis by the Eulerian viewpoint of ultrasonic wave velocity in the uniformly deformed isotropic solid," Journal of Materials Science, vol. 22, no. 5, pp. 1850-1856, 1987.

[12] J. A. Hudson, "Wave speeds and attenuation of elastic waves in material containing cracks," Journal of Royal Astronomical Society, vol. 64, no. 1, pp. 133-150, 1981.

[13] P. Shokouhi, R. Feldmann, and H. Wiggenhauser, "Stressdependency of sonic velocity in concrete under uniaxial load," in Proceedings of the 87th TRB Annual Meeting, Washington, DC, USA, 2008.

[14] A. Zoëga and H. Wiggenhauser, "Propagation time measurements of elastic surface waves on concrete specimens while under different loads and dependent to the direction of strain," in NDE/NDT for Highways and Bridges: Structural Materials Technology (SMT), ASNT, Oakland, Calif, USA, 2008.

[15] A. Zoëga, P. Shokouhi, and H. Wiggenhauser, "Propagation time of elastic surface waves on concrete specimens under uniaxial loads," Journal of Structural Engineering, vol. 36, no. 1, pp. 11-15, 2009. 

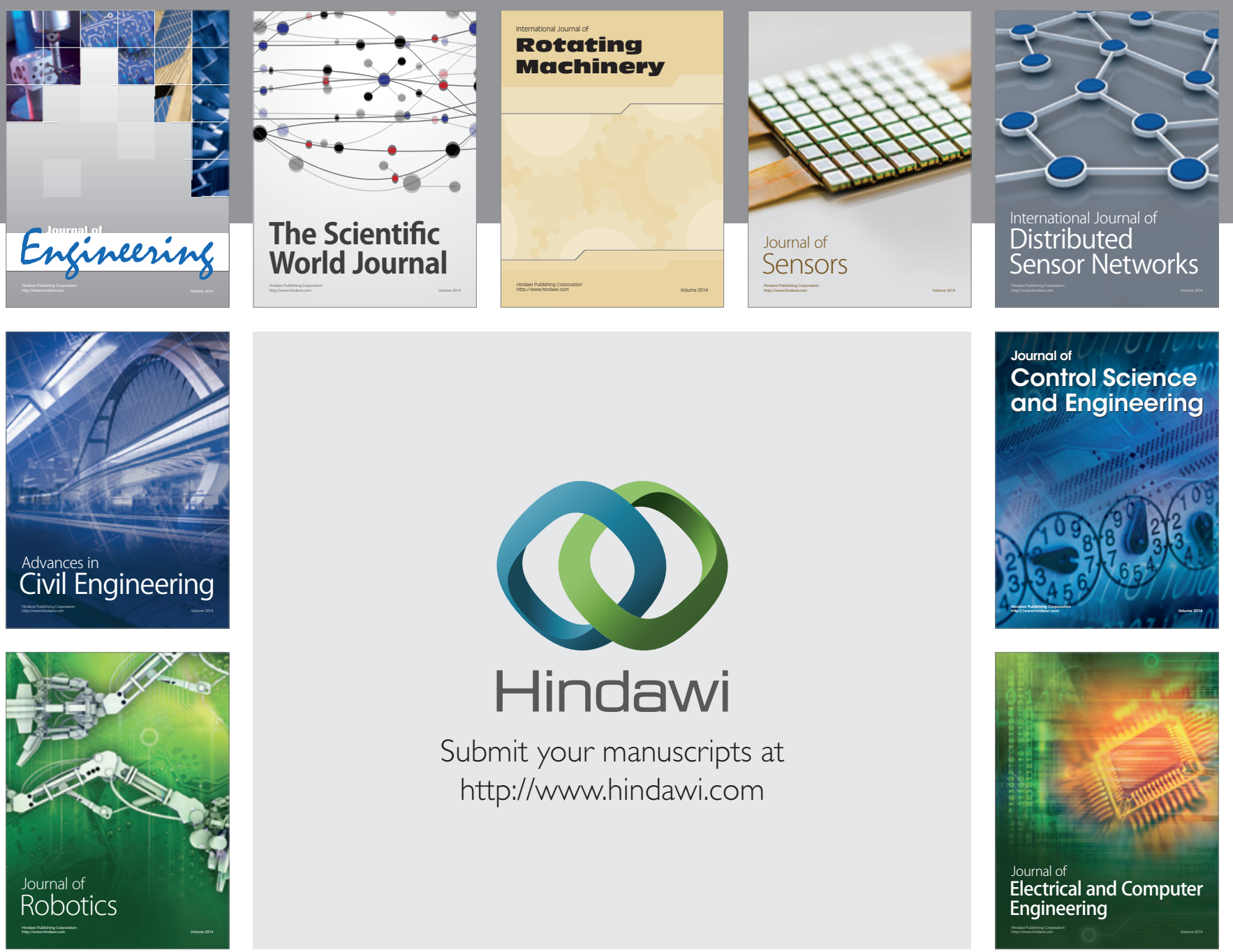

Submit your manuscripts at

http://www.hindawi.com
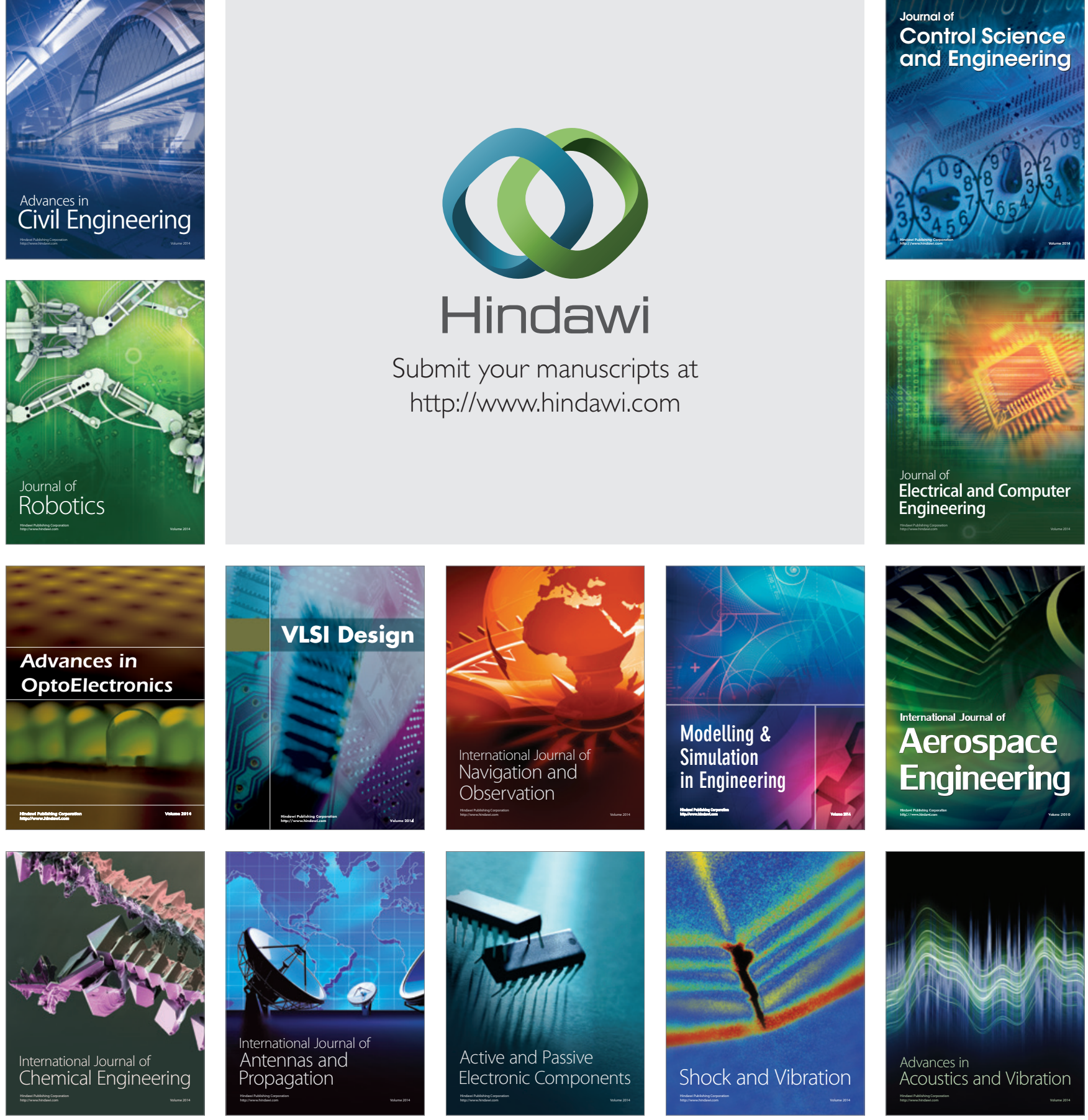